\title{
Correction to: effects of dietary gelatin hydrolysates on bone mineral density in magnesium-deficient rats
}

Teruyuki Noma ${ }^{1,2,3}$, Satoshi Takasugi ${ }^{1 *}$, Miho Shioyama' ${ }^{1}$, Taketo Yamaji ${ }^{1}$, Hiroyuki Itou' ${ }^{1}$, Yoshio Suzuki ${ }^{2}$ Keishoku Sakuraba ${ }^{2,3}$ and Keisuke Sawaki ${ }^{2}$

After the publication of this article [1] the authors pointed out to us that a formula was incorrectly shown due to an error during typesetting as:

$$
\begin{aligned}
& \text { Ultimate stress }=8 \mathrm{bFL} /\left(\mathrm{ab}^{3}-\mathrm{a}^{\prime} \mathrm{b}^{\prime} 3\right) \pi \\
& \text { Young s modulus }=4 \mathrm{FL}^{3} / 3 \mathrm{~d}\left(\mathrm{ab}^{3}-\mathrm{a}^{\prime} \mathrm{b}^{\prime} 3\right) \pi
\end{aligned}
$$

It should have been shown as:

$$
\begin{aligned}
& \text { Ultimate stress }=8 \mathrm{bFL} /\left(\mathrm{ab}^{3}-\mathrm{a}^{\prime} \mathrm{b}^{\prime 3}\right) \pi \\
& \text { Young s modulus }=4 \mathrm{FL}^{3} / 3 \mathrm{~d}\left(\mathrm{ab}^{3}-\mathrm{a}^{\prime} \mathrm{b}^{3}\right) \pi
\end{aligned}
$$

BMC apologise for this error. The original article was corrected.

\section{Author details}

'Division of Research and Development, Food Science Research Laboratories, Meiji Co., Ltd., 540 Naruda, Odawara, Kanagawa 250-0862, Japan. ${ }^{2}$ Graduate School of Health and Sports Science, Juntendo University, Chiba, Japan.

${ }^{3}$ Graduate School of Medicine, Juntendo University, Tokyo, Japan.

Received: 2 October 2017 Accepted: 6 October 2017

Published online: 24 October 2017

\section{Reference}

1. Noma, et al. Effects of dietary gelatin hydrolysates on bone mineral

density in magnesium-deficient rats. BMC Musculoskelet Disord. 2017;18:

385. doi:10.1186/s12891-017-1745-4.

\footnotetext{
*Correspondence: satoshi.takasugi@meiji.com

'Division of Research and Development, Food Science Research Laboratories,

Meiji Co., Ltd., 540 Naruda, Odawara, Kanagawa 250-0862, Japan

Full list of author information is available at the end of the article
} 